\title{
Quantum and Classical Ballistic Transport in Constricted Two-Dimensional Electron Gases
}

\author{
H. van Houten ${ }^{1}$, B.J. van Wees ${ }^{2}$, and C.W.J. Beenakker ${ }^{1}$ \\ ${ }^{1}$ Philips Research Laboratories, 5600 JA Eindhoven, The Netherlands \\ 2Delft University for Technology, 2600 GA Delft, The Netherlands
}

An experimental and theoretical study of transport in constricted geometries in the two-dimensional electron gas in high mobility GaAs-AJGaAs heterostructures is described. A discussion is given of the influence of boundary scattering on the quantum interference corrections to the classical Drude conductivity in a quasi-ballistic regime, where the transport is ballistic over the channel width, but diffusive over its length. Additionally, results are presented of a recent study of fully ballistic transport through quantum point contacts of variable width defined in the two dimensional electron gas. The zero field conductance of the point contacts is found to be quantized at integer multiples of $2 e^{2} / h$, and the injection of ballistic electrons in the two dimensional electron gas is demonstrated in a transverse electron focussing experiment.

\section{Introduction}

A variety of length scales governs the electron transport at low temperatures (see Table I). The mean free path $l_{\mathrm{c}}$ in a degenerate electron gas is determined by elastic scattering from stationary impurities. In homogeneous samples the classical transport can be described by the local Drude conductivity $\sigma_{D}=n e^{2} l / m v_{F}$, with $n$ the electron gas density, $v_{F}$ the Fermi velocity, and $m$ the electron effective mass. In a two-dimensional electron gas (2-DEG) this classical expression can also be written as $\sigma_{D}=\left(e^{2} / h\right) k f_{e}$, with $k_{F}=m v_{F} / h$ the Fermi wave vector. The Drude conductance $G_{D}$ of a 2-DEG channel of length $L$ and width $W$ is $G_{D}=(W / L) \sigma_{D}$. In two dimensions the smallest system for which a conductivity can be defined is a square of side of order $l_{l}$. The above expression for the conductance $G_{D}$ of a larger channel can thus be seen as the result of the classical addition of such small squares in a $\left(W / l_{e}\right)$ by $\left(L / l_{e}\right)$ array (see Fig. 1). It is clear that any correlation between the squares is neglected in this approach.

It is now well known that such correlations are important because of quantum interference [1]. Constructive interference betw'een time reversed backscattered electron haves leads to a conductivity decrease known as weak localization. Clearly, a prerequisite for this quantum interference effect is that the electrons maintain their phase coherence over a time $\tau_{\phi}$ long compared to the elastic scattering time $\tau_{f} \equiv l_{e} / v_{F}$.

\begin{tabular}{llll}
\hline Table I. Length scales for electron transport at low temperatures & \\
\hline elastic mean free path & $l_{c}=v_{F} \tau_{e}$ & Fermi wavelength & $\lambda_{F}=2 \pi / k_{F}$ \\
phase coherence length & $l_{\sigma}=\left(D \tau_{\phi}\right)^{1 / 2}$ & thermal length & $l_{T}=(h D / k T)^{1 / 2}$ \\
magnetic length & $l_{B}=(h / e B)^{1 / 2}$ & cyclotron radius & $l_{c}=h k_{F} / e B$
\end{tabular}




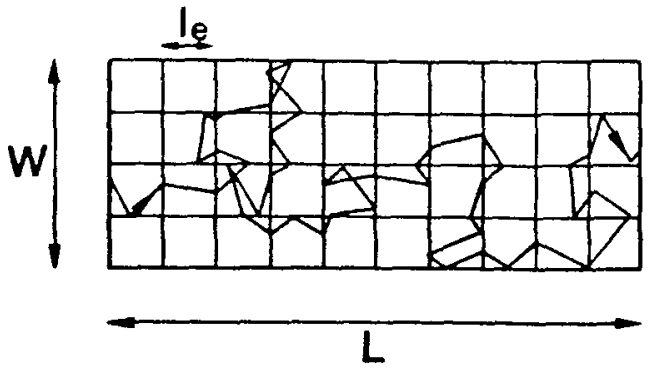

Fig. I Idealized picture of the Drude conductance; diffusive motion occurs on length scales large compared to $l_{e}$, and ballistic motion on shorter length scales.

The phase coherence length is a diffusion length given by $l_{\phi} \equiv\left(D \tau_{\phi}\right)^{1 / 2}$, with $D=v, \mu_{c} / 2$ the diffusion constant. The localization is one-dimensional (1D) if $l_{\phi}>W$. A second interference effect is the occurrence of universal (magneto) conductance fluctuations (UCF) in small samples, caused by interference of electrons on different trajectories [2]. This effect has two characteristic length scales, $l_{\phi}$ and the thermal length $l_{T} \equiv(h D / k T)^{1 / 2}$. From a beautiful series of experiments performed in the diffusive regime in metal rings [3] and in silicon MOSFETs [4] the quantum interference corrections to the Drude conductivity are known to be non-local on length scales smaller than $l_{\phi}$.

Classically, the simple scaling formula $G_{D}=(W / L) \sigma_{D}$ breaks down on length scales short compared to the mean free path. In this paper we distinguish a quasi-ballistic regime $\left(L \gg I_{r}>W\right)$, where the electrons move ballistically over the sample width, but diffusively over its length, and a fully ballistic regime $\left(l_{r} \gg W, L\right)$. The scattering of the electrons by typical smooth electron gas boundaries is specular, because of the large Fermi wave length $\left(\lambda_{F} \sim 40 \mathrm{~nm}\right)$. We note that the presence of voltage probes on the channel sides will in general have an inextricable influence on the electron transport [5]. We will here concentrate on channels which are smooth constrictions connected by broad 2-DEG regions to ohmic contacts (see Fig. 2). While in the quasi-ballistic regime the measured conductance is an intrinsic property of the narrow channel, this is no longer the case in the ballistic regime. Here the channel does not have an intrinsic resistance and, as we will discuss below, the conductance is determined by a geomet-
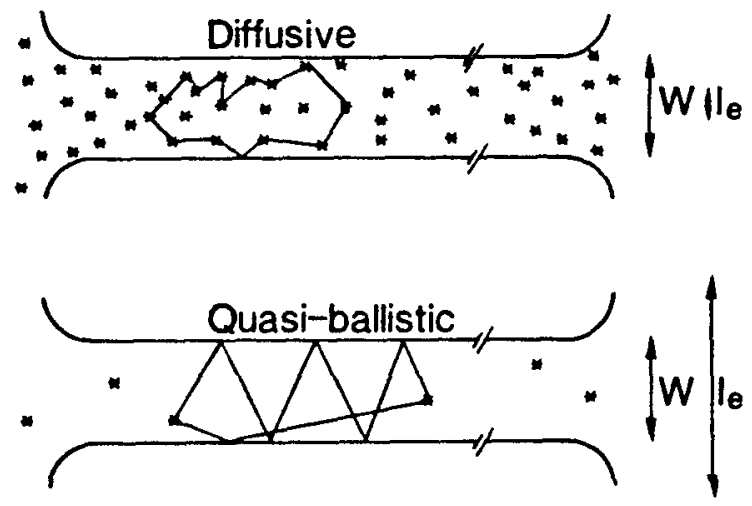

Fig. 2 Transport in constricted regions of length $L$ and width $W$ for three mobility regimes. Closed trajectories, necessary for weak localization, occur

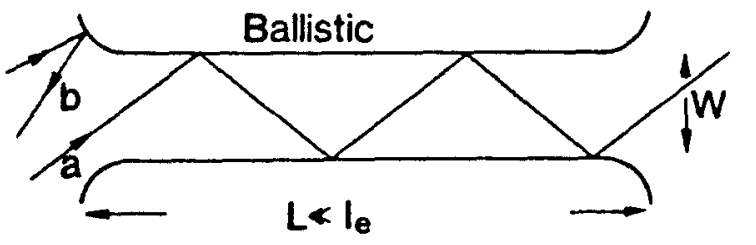
in the diffusive and quasi-ballistic regimes. Only' open (a) trajectories occur in the ballistic regime, where the resistance is a geometrical constriction effect, associated with trajectories of type (b). 
rical constriction effect. If $\lambda_{F}$ is comparable to the constriction width it is possible to study quantum ballistic transport.

In the quasi-ballistic regime we have experimentally [6] and theoretically [7] investigated weak localization and UCF in long narrow channels defined in moderately high mobility GaAs-AlGaAs heterostructures. In the first part of this paper we give a qualitative account of the effect of a magnetic field on these quantum interference effects in narrow channels for various mobility regimes. An unsolved problem is presented by the breakdown of coherent diffusion on timescales shorter than $\tau_{p}$, which is important if $\tau_{\phi}$ and $\tau_{e}$ are comparable.

In the fully ballistic regime disorder related quantum interference effects are absent. In metal physics Sharvin [8] point contacts (i.e. constrictions much narrower and shorter than the mean free path) can ideally be used to study ballistic transport. We have very recently succeeded in fabricating such point contacts in the 2-DEG of high mobility GaAs-AlGaAs heterostructures $[9,10]$. A split-gate lateral depletion technique allows the width of the constrictions to be continuously varied (between 0 and $200 \mathrm{~nm})$. We have found that the conductance of these quantum point contacts exhibits quantized plateaux at integer multiples of $2 e^{2} / h$ if their width is varied. An explanation of this novel effect is given in terms of the quantization of the transverse electron momentum in the narrow part of the constriction. A semi-classical treatment of the quantized conductance in such ballistic point contacts is presented and, alternatively, it is discussed in the context of the Landauer formula [16].

Finally, the existence of skipping orbits of electrons in the 2-DEG is demonstrated in a transverse electron focussing experiment. Here electrons are injected by one point contact and, after deflection by a magnetic field and repeated reflections on a 2-DEG boundary, they are collected by a second point contact. Finestructure in the focussing spectra, attributed to interference of the ballistic electrons, is resolved at low temperatures.

\section{Flux Cancellation Effect on Weak Localization and UCF}

Weak localization originates in the constructive interference between a closed electron trajectory and its time reverse. A magnetic field destroys this effect, leading to a negative magnetoresistance. A semi-classical treatment in terms of electron trajectories is allowed for channels much wider than the Fermi wavelength. The ID-weak localization conductance correction can be expressed as a time integral over the classical probability of return $C(t)[12]$

$$
\delta G_{l o c}(B)=-\frac{4 e^{2}}{h} \frac{D}{L} \int_{0}^{\infty} d t C(t) e^{-t / \tau} \phi<e^{i \phi(t)}>.
$$

The factor $\exp \left(-t / \tau_{\phi}\right)$ accounts for the fact that only electrons which have retained their phase coherence over a time $t$ can interfere constructively. In a magnetic field a phase difference $\phi(t)$ develops between time reversed trajectories. This effect is taken into account by the factor $\langle\exp i \phi\rangle$. The brackets denote an average over impurity configurations. For weak magnetic fields this factor decays as $\exp \left(-t / \tau_{B}\right)$ (see Ref. [7]). The meaning of the relaxation time $\tau_{B}$ is that, in the presence of a magnetic field $B$, Irajectories with a duration $t$ exceeding $\tau_{B}$ no longer contribute to the localization. On length scales larger than $l_{\text {, the }}$ probability density of return is given by $C(t)=(4 \pi D t)^{-1,2}$. One thus finds [1]

$$
\delta G_{l o c}(B)=-\frac{2 e^{2}}{h} \frac{\sqrt{D}}{L}\left(\frac{1}{\tau_{\phi}}+\frac{1}{\tau_{B}}\right)^{-1 / 2} .
$$


We define a critical field $B^{*}$ for the suppression of weak localization as the field for which $\tau_{\phi}=\tau_{B}$. The relaxation time $\tau_{B}$ in a narrow channel with specular boundary scattering has been calculated in Ref. [7] , for the quasi-ballistic regime (see Refs. [13 ... 15] for other regimes). In Table Il the main results of the analysis are summarized. In this section we present a simple physical interpretation of these results.

Table II. Magnetic field phase relaxation time $\tau_{B}$ and critical field $B^{\circ}$ for $1 D$-weak localization $[7,13]\left(C_{1}=9.5\right.$ and $\left.C_{2}=24 / 5\right)$. In the quasi-ballistic regime the critical field is enhanced as a consequence of the flux cancellation effect.

Diffusive $\left(l_{e} \ll W, L\right) \quad$ Quasi-ballistic $\left(W \ll l_{e} \ll L\right)$

ID $l_{B}^{2} \gg W^{2} \quad$ Weak field $l_{B}^{2} \gg W l_{c} \quad$ Strong field $W l_{c} \gg l_{B}^{2} \gg W^{2}$

$$
\begin{array}{lll}
\tau_{B}=\frac{6 l_{B}^{4}}{W^{2} v_{F} J_{e}} & \tau_{B}^{\text {weak }}=\frac{C_{1} l_{B}^{4}}{W^{3} v_{F}} & \tau_{B}^{\text {strong }}=\frac{C_{2} l_{B}^{2} l_{e}}{W^{2} v_{F}} \\
\dot{B}=\frac{h}{e} \frac{\sqrt{3}}{W l_{\phi}} & \dot{B}=\frac{h}{e} \frac{1}{W l_{\phi}} \sqrt{\frac{C_{1} l_{e}}{2 W}} & B=\frac{h}{e} \frac{1}{W l_{\phi}} \frac{C_{2} l_{e}^{2}}{2 W l_{\phi}}
\end{array}
$$

The effectiveness of a magnetic field in suppressing weak localization depends in an interesting way on the shape of the electron trajectories. Three regimes can be distinguished, depending on the relative magnitudes of $l_{e}, W$, and the magnetic length $l_{B} \equiv(h / e B)^{1 / 2}$. This is illustrated in Fig. 3 , where the relaxation time $\tau_{B}$ is plotted as a function of $l_{C} / W$ for a fixed ratio $l_{B} / W$. The non-monotonous dependence of $\tau_{B}$ on $l_{C}$ can be understood as follows.

In the diffusive regime $\left(l_{e} \ll W, L\right)$ an increase of the mean free path is seen to lead to a decrease of $\tau_{B}$. The phase change for a closed loop is given by the enclosed flux divided by $h / e$, or equivalently, by its atea divided by $l_{B}^{*}$. In the diffusive regime the enclosed area for a trajectory of duration $\tau_{B}$ is of order $W\left(D \tau_{B}\right)^{1 / 2}$. Setting this area equal to $l_{B}^{2}$ one finds (apart from numerical coefficients) the diffusive regime result for

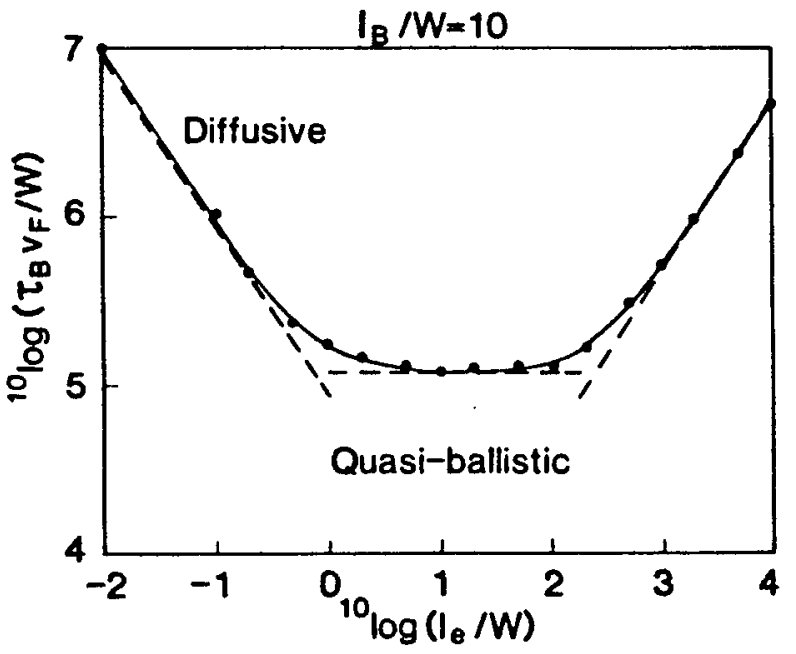

Fig. 3 Phase relaxation time $\tau_{B}$ as a function of the elastic mean free path $l_{e}$. The plot is obtained by a numerical calculation $[7]$ with $l_{B}^{\circ}=10 \mathrm{~W}$. Dashed lines are analytic results (see Table II) The results show the characteristic dependence of $\tau_{B}$ on $l_{c}$ in, respectively, the diffusive and quasi-ballistic regime. The latter regime can be subdivided in a weak $\left(W l_{e} l_{B}^{2} \ll 1\right)$ and a strong $\left(W l_{e} \mid l_{B}^{2} \gg 1\right)$ field regime. 
$\tau_{B}$ listed in Table II. The decrease of $\tau_{B}$ on increasing $l_{f}$ is thus simply a consequence of the faster diffusion, leading to a larger enclosed area for a given duration $\left(D=v_{F} / 2\right)$.

On further increasing the mean free path the quasi-ballistic regime is reached. In this regime frequent collisions with the boundary lead to a crossing of the returning electron trajectories. Since the various closed parts of such trajectories are traversed in opposite directions the net enclosed flux is greatly reduced (flux cancellation effect) [16]. This effect is illustrated in the inset of Fig. 4. A weak and strong field regime have to be distinguished, depending on the ratio $W l_{e} / l_{B}^{2}$. This ratio corresponds to the maximum phase change on a closed trajectory of linear extension $l_{i}$.

In the weak field regime $\left(W l_{d} / l_{B}^{2} \ll 1\right)$, many impurity collisions are needed before the electron loop encloses sufficient flux for a complete phase relaxation. In this regime a further increase of the mean free path does not lead to a larger enclosed flux, because the effect of the larger loop area is compensated by the increased flux cancellation. Consequently $\tau_{B}$ in the plot of Fig. 3 is approximately constant. On comparing the result for $B$ in the weak field regime with the result for the diffusive regime, we see an enhancement of the critical field by a factor $\left(l_{e} / W\right)^{1 / 2}$ (see Table II).

The strong field regime is reached if $W l_{e} / l_{B}^{2} \gg 1$ (note that the theory only applies for fields limited by the condition $l_{B} \gg W$ ). Under these conditions, trajectories involving a single glancing angle reflection from the channel boundary enclose sufficient flux. In this regime the phase relaxation for trajectories of a given duration $t$ is no longer determined by the average linear extension, but by the probability for these glancing angle reflections to occur. This probability is proportional to the number of impurity collisions, and thus to $1 / l_{e}$. The relaxation time $\tau_{B}$ accordingly increases with $l_{e}$ in this regime (see Fig. 3).

As seen in Fig. 3 the results given in Table II for the various asymptotic regimes agree very well with the results of a numerical calculation. For comparison with experiments in the quasi-ballistic regime the following simple interpolation formula can be used:

$$
\tau_{B}=\tau_{B}^{\text {wedh }}+\tau_{B}^{\text {strong }},
$$

with $\tau_{B}^{\text {wedk }}$ and $\tau_{B}^{\text {strong }}$ as given in Table II. So far we have assumed that the transport is diffusive on time scales corresponding to $\tau_{\phi}$. This will be a good approximation only if $\tau_{\phi} \gg \tau_{p}$. As we have discussed elsewhere [6], a modification of Eq. (1) is necessary to take the breakdown of coherent diffusion into account in the case that $\tau_{\phi}$ and $\tau_{p}$ are comparable. We note that for very high mobility channels, with smooth boundaries, the weak localization effect vanishes, because the ballistic electron motion prevents the backscattering needed for this effect (see Fig. 2).

We now turn to the related problem of the magnetoconductance fluctuations observed in small samples. From the experimental data a conductance auto correlation function $F(\Delta B)=\langle\delta G(B) \delta G(B+\Delta B)\rangle$ with $\delta G(B)=G(B)-\langle G(B)>$ can be determined. Here the average is over magnetic field values [2]. The field increment $\Delta B_{c}$ such that $F\left(\Delta B_{1}\right)=F(0) / 2$ is by definition the correlation field, while $F(0)$ represents the variance. At $T=0$ the variance $F(0)$ is of order $\left(e^{2} / h\right)^{2}$. At finite temperatures in quasi-one dimensional channels $\left(W \ll l_{\phi} \ll L\right), F(0)$ can be approximated by [7]

$$
F(0)=6\left(\frac{e^{2}}{h}\right)^{2}\left(\frac{l_{\phi}}{L}\right)^{3}\left[1+\frac{9}{2 \pi}\left(\frac{l_{\phi}}{l_{T}}\right)^{2}\right]^{-1} \text {. }
$$

Here $l_{T}$ accounts for the non-monochromaticity of the electrons associated with the thermal smearing of the Fermi-Dirac distribution [2]. This effect is of importance only if $l_{T}<l_{\phi}$. On length scales $L$ larger than $l_{\phi}$ the relative magnitude of the fluctu- 
ations $F(0)^{1 / 4} / G_{D}$ decreases as $L^{-1 / 2}$ as a consequence of the classical averaging over uncorrelated segments of length $l_{\phi}$. (For comparison we note that $\delta G_{l o s} / G_{D}$ does not depend on $L$ if $L \gg l_{\phi}$ ). The expression (4) for the variance applies to the diffusive and quasi-ballistic regimes, under the assumption of coherent diffusion $\left(\tau_{e} \ll \tau_{\phi}\right)$. As mentioned earlier in the context of weak localization, these disorder related quantum interference effects vanish for purely ballistic electron motion.

The correlation field $\Delta B_{c}$ depends on the shape of the trajectories (via the enclosed flux), and this is where boundary scattering plays an essential role. Even though the type of trajectories. involved differs from the closed trajectories responsible for weak lecalization, the problem of determining $\Delta B_{c}$ is essentially the same as the one for $B$ discussed before. As a typical example the theoretical results [7] for the correlation field are plotted in Fig. 4 for the diffusive and quasi-ballistic regimes' (for the case $\left.l_{T} \gg l_{\phi}\right)$. This figure clearly shows the enhancement of the correlation field as a consequence of the flux cancellation effect in the quasi-ballistic regime.

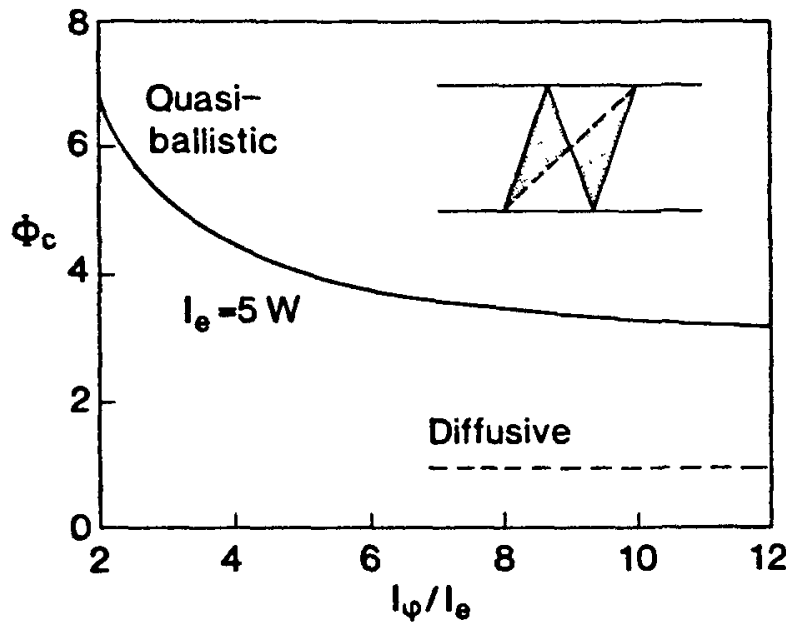

Fig. 4 Plot of the correlation flux $\phi_{c} \equiv \Delta B_{c} / l_{\phi} W$ (in units of $h / e$ ) for Universal Conductance Fluctuations as a function of the phase coherence length $l_{\phi}$ (normalized by the elastic mean free path) in the quasi-ballistic and diffusive regime. The inset illustrates the characteristic flux cancellation: the shaded areas are equal and of opposite orientation.

\section{Quantum Point Contacts}

The conductance of ballistic, or Sharvin, point contacts is purely determined by its geometry, and by the electron momentum at the Fermi level $h k_{F}$. For simplicity we consider the equivalent (by the Einstein relation) transport problem of uncharged particles [11], where the current arises as a consequence of the concentration gradient or chemical potential difference $\Delta \mu$ across the constriction. The electron distribution in $\mathbf{k}$-space in the constricted region is schematically drawn in Fig. 5 . The current $I$ passing through a constriction of length $L$ and width $W$ equals the number of excess carriers present in the constriction $(W L) N_{0} \Delta \mu / 2$, divided by the transit time $L / v_{F} \cos \phi$. Here $N_{o}=m / \pi h^{2}$ is the 2-DEG areal density of states (assuming spindegeneracy). The conductance $G \equiv J e^{2} / \Delta \mu$ is thus $G=(1 / 2) e^{2} N_{0} W\left\langle v_{F} \cos \phi>\right.$, where the brackets denote an angular average over positive values of $\cos \phi$. .Classically, all values of $\phi$ have equal weight, leading to a conductance [9]

$$
G=\frac{2 e^{2}}{h} \frac{k_{f} W}{\pi},
$$

proportional to the constriction width and the Fermi momentum.

We have recently fabricated the first ballistic point contacts in the 2-DE் in high mobility GaAs-AlGaAs heterostructures $[9,10]$. A split-gate lateral depletion tech- 


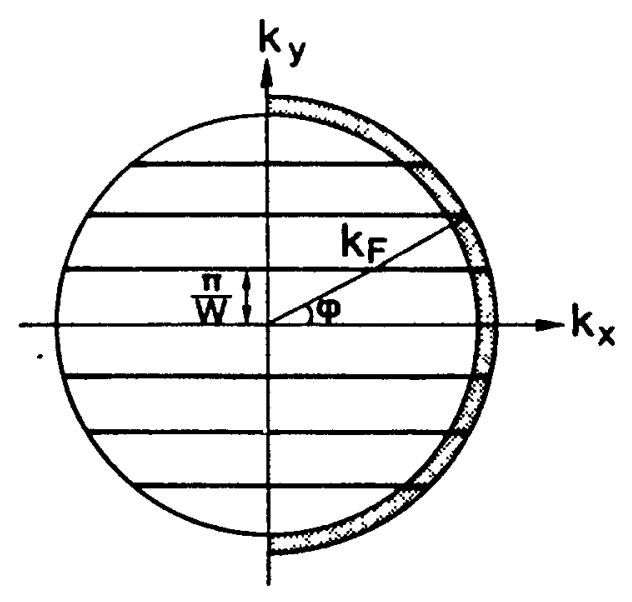

Fig. 5 Electron distribution in k-space in a constricted 2-DEG region. The current in the $x$-direction is carried by ballistic electrons in the shaded area. Quasi-one-dimensional electric subbands (horizontal lines), lead to the quantized conductance of Fig. 6.

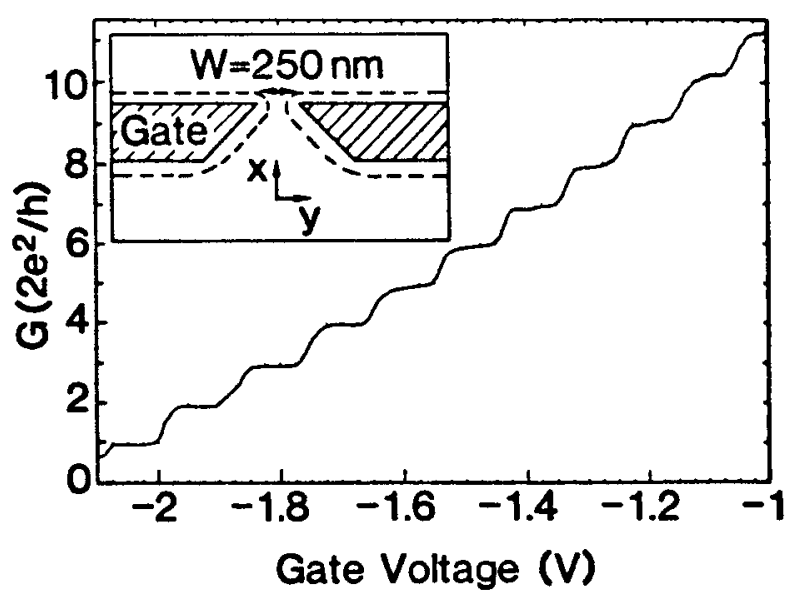

Fig. 6 Conductance of a ballistic quantum point contact at $0.6 \mathrm{~K}$ as a function of gate voltage in the absence of a magnetic field. The inset shows the sample geometry. The dashed line schematically indicates the 2-DEG boundary. Note that the point contact is actually a constriction of finite length.

nique [17] is used to vary the constriction width continuously. On varying the gatevoltage we have found the two-terminal conductance of these ballistic point contacts to exhibit quantized plateaux at integer multiples of $2 e^{2} / h$ in the absence of a magnetic field (see Fig. 6) [18]. This novel effect can be understood as a consequence of the quantization of the transverse electron momentum in the narrow region, whereby only discrete values for the angle $\phi$ are allowed (see Fig. (5)). These values correspond to one-dimensional electric subbands with $k_{b}= \pm n \pi / W, n=1,2, \ldots N_{c}$; the total number of subbands (or channels) $N_{c}$ is the largest integer smaller than $k_{F} W / \pi$ (for a square well lateral confining potential). Each subband carries an equal amount of current, because the loss in forward momentum for large $n$ values is compensated by an increase of the ID density of states. From the classical correspondence with Eq. (5) it thus follows that $G=\left(2 c^{2} / h\right) N_{c}$. In the actual experiment a local change in electron gas density $n_{s}=k_{F}^{2} / 2 \pi$ accompanies a change in constriction width. We note that these changes have a similar effect on the number of subbands, and thus on the conductance. In order for the preceding theoretical arguments to apply one needs a channel longer than wide, so that life time broadening of the $1 \mathrm{D}$ subbands in the constriction is unimportant. This assumption appears to be validated by our experiment.

The above semi-classical approach can be substantiated by the quantum mechanical Landauer formula $[11,19]$

$$
G=\frac{2 e^{2}}{h} N_{c}(1-r) \text {, }
$$

where $r$ is the fraction of electrons injected in the constriction which scatter back into the wide 2DEG regions. Originally, this type of formula was derived to treat a disordered region connected by means of ideal leads to thermalizing reservoirs, in which all inelastic scattering events are thought to take place exclusively. Apparently, quantum point contacts provide a model system which closely approximates such an idealized 
wire, in which the absence of a disordered region leads to $r=0$, and thus to $G=N_{c}\left(2 e^{2} / h\right)$.

We now digress to discuss a more general applicability of the formula $G=N_{c}\left(2 e^{2} / h\right)$ to ballistic transport. First of all, the two-terminal conductance of a 2-DEG in the quantum Hall regime is given by $G=N_{L}\left(2 e^{2} / h\right)$, with $N_{L}$ the number of occupied Landau levels (still assuming spin degeneracy). This number is given by $N_{L}=E_{F} / h \omega_{c}=k_{F} l_{c} / 2$, with $l_{c}=m v_{F} / e B$ the classical cyclotron radius. This number of Landau levels equals the number of quantum edge states at the Fermi energy [20], which are localized within a distance $2 l_{c}$ from the electron gas boundary. These edge states take the place of the 1D electric subbands which, as explained above, cause the quantized conductance of ballistic point contacts in the absence of a magnetic field. The above point of view has been confirmed very recently by experiments showing that the quantization of the two-terminal conductance of ballistic point contacts is preserved in a magnetic field, the only change being that $N_{c}$ is replaced by the number of occupied magneto - electric subbands [21] in the constricted region [22] .

Finally, we remark that under certain conditions four-terminal measurements in the ballistic regime can also be understood in terms of the preceding considerations. The four-terminal resistance measured over a constriction is then $R_{A_{1}}=\left(h / 2 e^{2}\right)\left(N_{1}^{-1}-N_{2}^{-1}\right)$, with $N_{1}$ the number of subbands in the constriction and $N_{2}$ the number of Landau levels in wide regions near the voltage probes. This formula has been used to describe the suppression of the Sharvin contact resistance by a magnetic field in a four-terminal set-up [23].

\section{Coherent electron focussing}

In 1965, Sharvin proposed a method to study Fermi surfaces in metals, in which ballistic electrons injected by a point contact are focussed by a longitudinal magnetic field onto a second, collecting, point contact [8]. We have performed [10] an electron focussing experiment in a 2-DEG in the transverse field geometry [24]. The experimental arrangement is shown in Fig. 7. In this geometry the scattering of the injected electrons by the 2-DEG boundary can be studied by comparing the intensities of the focussing peaks associated with multiple reflections, or skipping orbits, of the electrons. The experimental results, shown in Fig. 8, clearly establish that injection of ballistic electrons is realized in this experiment. The large number of classical focussing peaks observed (at $4 \mathrm{~K}$ ) proves that the scattering at the 2-DEG boundaries indeed is highly specular, as we have assumed thoughout this paper.

At low temperatures interesting finestructure is apparent in the focussing spectra. We believe that this is caused by interference of electrons on different skipping orbits from injector to collector. The experiment thus reflects the spatial coherence of the injected ballistic electrons. We are currently studying a quantitative model for this

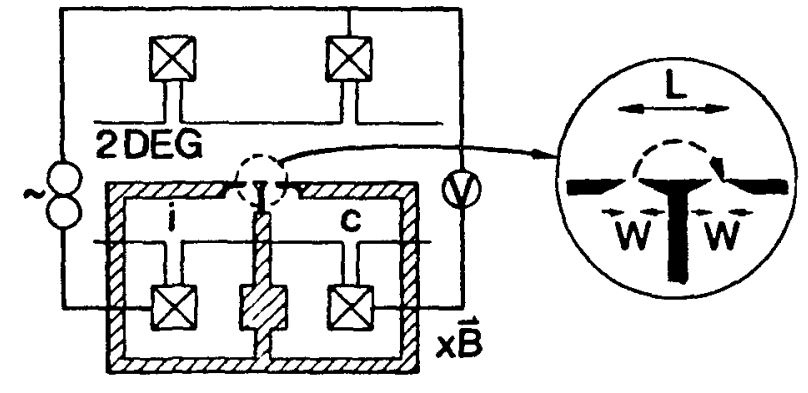

Fig. 7 Layout of the split-gate (shaded area) double point contact structure for transverse electron focussing experiments in a 2-DEG. Crossed squares are ohmic contacts. The gate separates injector (i) and collector (c) areas from the bulk 2-DEG. The dashed line indicates an electron trajectory in a magnetic field. 


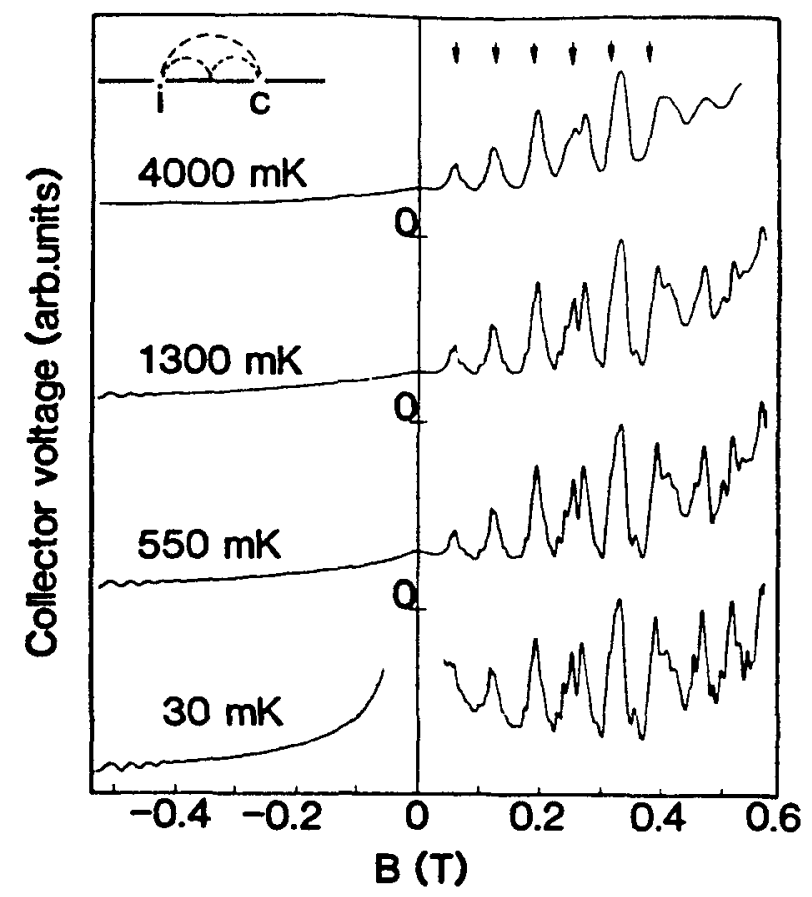

Fig. 8 Transverse electron focussing spectra showing classical, equidistant, focussing peaks at $4 \mathrm{~K}$ and fine structure at low temperatures. Calculated positions of the classical focussing peaks are indicated by arrows. The inset illustrates typical trajectories, corresponding to the first and second maxima.

effect. We conclude by noting that the transverse electron focussing geometry of Fig. 7 offers excellent opportunities to study the precise role of the coupling of voltage probes to quantum conductance channels. This is especially so in view of the fact that a point contact may be the least disturbing voltage probe $[11,19]$ which can be realized with the present state of the art.

Valuable contributions to the work described in this paper have been made by M.E.I. Broekaart, L.P. Kouwenhoven, J.M. Lagemaat, P.H.M. van Loosdrecht, J.E. Mooij, J.A. Pals, M.F.H. Schuurmans, C.E. Timmering and J.G. Williamson. The high mobility samples needed for this work were grow'n by C.T. Foxon and J.J. Harris.

\section{References}

1. See the review by P.A. Lee and T.V. Ramakrishnan, Rev. Mod. Phys. 57 (1985) 287.

2. B.L. Al'tshuler, Pis'ma Zh.Eksp. Teor. Fiz. 41 (1985) 530 (JETP Lett., 41 (1985) 648); P.A. Lee, A. D. Stone and H. Fukuyama, Phys. Rev. B. 35 (1987) 1039.

3. C.P. Umbach, P. Santhanam, C. van Haesendonck and R.A. Webb, Appl. Phys. Lett., 50 (1987) 1289;

4. W.J. Skocpol, P.M. Mankicwich, R.E. Howard, L.D. Jackel, D.M. Tennant and A.D. Stone, Phys. Rev. Lett. 58 (1987) 2347.

5. G. Timp, A.M. Chang, P. Mankiewich, R. Behringer, J.E. Cunningham, T.Y. Chang and R.E. Howard, Phys. Rev. Lett. 59 (1987) 732; M.L. Roukes, A. Scherer, S.J. Allen Jr., H.G. Craighead, R.M. Ruthen, E.D. Beebe and J.P. Harbison, Phys. Rev. Lett. 59 (1987) 3011.

6. H. van Houten, C.W.J. Beenakker, B.J. van Wees and J.E. Mooij, Surf. Sci. 196, (1988) 144; H. van Houten, C.W.J. Bcenakker, M.E.I. Broekaart, M.G.J. Heijmans, B.J. van Wees, J.E. Mooij and J.P. André, Acta Electronica, in press.

7. C.W.J. Beenakker and H. van Houten, Phys. Rev. B., in press.

8. Y. V. Sharvin, Zh. Eksp. Teor. Fiz. 48 (1965) 984 (Sov. Phys.-JETP 21 (1965) 655). 
9. B.J. van Wees, H. van Houten, C.W.J. Becnakker, J.G. Williamson, L.P. Kouwenhoven, D. van der Marel and C.T. Foxon, Phys. Rev. Lett. 60 (1988) 848.

10. H. van Houten, B.J. van Wees, J.E. Mooij, C.W.J. Beenakker, J.G. Williamson, C.T. Foxon, Europhys. Lett., in press.

11. R. Landauer, I.B.M. J. Res. Dev. 1 (1957) 223; Z. Phys. B 68 (1987) 217.

12. S. Chakravarty and A. Schmid, Physics Reports 140 (1986) 193.

13. B.L. Al'tshuler and A.G. Aronov, Pis'ma Zh. Eskp. Tcor. Fiz. 33 (1981) 515 (JETP Lett. 33 (1981) 499).

14. V.K. Dugaev and D.E. Khmel'nitskii, Zh. Eksp. Teor. Fiz. 86 (1984) 1784. (Sov. Phys. JETP 59 (1984) 1038);

15. See also R. Landauer and M. Büttiker, Phys. Rev. B. 36 (1987) 6255.

16. This phenomenon is also encountered in thin supcrconducting films, P.G. de Gennes and M. Tinkham, Physics (New York), 1 (1964) 107;

17. T.J. Thornton, M. Pepper, H. Ahmed, D. Andrews and G.J. Davies, Phys. Rev. Letters, 56 (1986) 1198; H.Z. Zheng, H.P. Wei and D.C. Tsui, Phys. Rev. B 34 (1986) 5635 .

18. Similar experimental results were obtained by D.A. Wharam, T.J. Thornton, R. Newbury, M. Pepper, H. Ahmed, J.E.F. Frost, D.G. Hasko, D.C. Peacock, D.A. Ritchie, G.A.C. Jones, J. Phys. C, to be published.

19. Sharvin contact resistances have been mentioned in the context of theoretical discussions of idealized mesoscopic systems in M. Buittiker, Phys. Rev. B. 33 (1986) 3020; and Phys. Rev. B, 35 (1987) 4123; Y. Imry in Directions in Condensed Matter Physics, Vol. 1, ed. G. Grinstein and G. Mazenko, World Scientific, Singaporc (1986) 102; See also B. van de Leemput, University of Nijmegen, The Netherlands, Master Thesis.

20. B.I. Halperin, Phys. Rev. B 25 (1982) 2185; P. Streda, J. Kucera and A.H. MacDonald, Phys. Rev. Lett. 59 (1987) 1973.

21. K.-F. Berggren, T.J. Thornton, D.J. Newson and M. Pepper, Phys. Rev. Lett. 57 (1986) 1769; H. van Houten, B.J. van Wees, J.E. Mooij, G. Roos and K.-F. Berggren, Superlattices and Microstructures, 3, (1987) 497.

22. B.J. van Wees, L.P. Kouwenhoven, H. van Houten, C.W.J. Becnakker, J.E. Mooij, C.T. Foxon and J.J. Harris, subm. to Phys. Rev. Lett.

23. H. van Houten, C.W.J. Beenakker, P.H.M. van Loosdrecht, T.J. Thornton, H. Ahmed, M. Pepper, C.T. Foxon and J.J. Harris, subm. to Phys. Rev. B.

24. V.S. Tsoi, ZhETF Pis. Red. 19 (1974) 114 (JETP Lett. 19 (1974) 70). 